\title{
Rotational symmetries of crystals with defects
}

\author{
G. P. Parry
}

Received: date / Accepted: date

Abstract I use the theory of Lie groups/algebras to discuss the symmetries of crystals with uniform distributions of defects.

Keywords Crystals $\cdot$ defects $\cdot$ Lie groups

\section{Introduction}

The rotational symmetries of perfect crystals are the focus of the theory of crystallography, and the most basic ideas in that theory are as follows. Let $L$ be a perfect lattice in $\mathbb{R}^{3}$, generated by basis vectors $\mathbf{e}_{1}, \mathbf{e}_{2}, \mathbf{e}_{3}$, so

$$
L \equiv\left\{\mathbf{x}: \mathbf{x}=n_{a} \mathbf{e}_{a}, \quad n_{a} \in \mathbb{Z}, \quad a=1,2,3\right\},
$$

where the summation convention operates on repeated indices. Note that the choice of basis vectors is not unique; indeed, if $\mathbf{e}_{1}^{\prime}, \mathbf{e}_{2}^{\prime}, \mathbf{e}_{3}^{\prime}$ is also a set of basis vectors for the lattice $L$, then there exists a matrix $\gamma \in G L_{3}(\mathbb{Z})$, with entries $\gamma_{a b}, a, b=1,2,3$, such that

$$
\mathbf{e}_{a}^{\prime}=\gamma_{a b} \mathbf{e}_{b}
$$

$\left(\gamma \in G L_{3}(\mathbb{Z})\right.$ if and only if each entry $\gamma_{a b}$ is an integer, and $\gamma^{-1}$ exists, also with each entry an integer. This implies that the determinant of $\gamma$ is either +1 or -1 .) Then, if $R$ is an orthogonal transformation of $\mathbb{R}^{3}$ to itself, that rotation maps $L$ to itself if and only if there exists $\gamma \in G L_{3}(\mathbb{Z})$ such that

$$
R \mathbf{e}_{a}=\gamma_{a b} \mathbf{e}_{b},
$$

where indices in (3) range from 1 to 3 , and this convention will be understood henceforward.

G. P. Parry

School of Mathematical Sciences, University of Nottingham, Nottingham NG7 2RD.

Tel.: +115-95-13845

Fax: +115-95-13837

E-mail: gareth.parry@nottingham.ac.uk 
If the basis $\mathbf{e}_{1}, \mathbf{e}_{2}, \mathbf{e}_{3}$ is given, the set of orthogonal transformations $R$ such that (3) holds for some $\gamma \in G L_{3}(\mathbb{Z})$ is the point group of $L$, denoted $P_{p}$, where the subscript $p$ denotes perfect crystal, and the set of matrices $\gamma \in G L_{3}(\mathbb{Z})$ such that (3) holds for some orthogonal transformation $R$ is the lattice group of $L$ relative to the given basis, denoted $\tilde{L}_{p}$. Note that $\tilde{L}_{p}$ depends on the choice of basis, whereas $P_{p}$ does not. From (3)

$$
R=\gamma_{a b} \mathbf{e}_{b} \otimes \mathbf{f}_{a},
$$

where the set of vectors $\mathbf{f}_{1}, \mathbf{f}_{2}, \mathbf{f}_{3}$ is dual to the basis $\mathbf{e}_{1}, \mathbf{e}_{2}, \mathbf{e}_{3}$ in the sense that

$$
\mathbf{e}_{a} \cdot \mathbf{f}_{b}=\delta_{a b},
$$

where $\delta_{a b}$ are the elements of the Kronecker delta. The groups $P_{p}, \tilde{L}_{p}$ are dual to each other in the sense that elements of the two groups are related via (4).

In this paper I show that this procedure may be generalized to account for crystals with uniform distributions of defects. To do so I confine attention to crystals whose 'texture' at a point $\mathbf{x} \in \mathbb{R}^{3}$ is defined by the values $\boldsymbol{\ell}_{a}(\mathbf{x}), a=1,2,3$, of three smooth 'lattice vector fields' $\ell_{1}(\cdot), \ell_{2}(\cdot), \ell_{3}(\cdot)$, which are linearly independent at each point cf. Davini [1]. This distribution of vector fields is uniform in the sense that the corresponding dislocation density tensor is constant (see later for precise definitions). In this case, it turns out that one can introduce a Lie group structure such that the lattice vector fields are right invariant with respect to the group multiplication - in continuum mechanical terms this means that there is an elastic deformation $\boldsymbol{\psi}=\boldsymbol{\psi}(\mathbf{x}, \mathbf{u})$, depending on a parameter $\mathbf{u}$, such that

$$
\ell_{a}(\boldsymbol{\psi}(\mathbf{x}, \mathbf{u}))=\nabla_{1} \boldsymbol{\psi}(\mathbf{x}, \mathbf{u}) \ell_{a}(\mathbf{x})
$$

where $\nabla_{1}$ denotes the (deformation) gradient with respect to the first argument. This elastic deformation is such that, in particular,

$$
\boldsymbol{\psi}(\boldsymbol{\psi}(\mathbf{x}, \mathbf{u}), \mathbf{w})=\boldsymbol{\psi}(\mathbf{x}, \boldsymbol{\psi}(\mathbf{u}, \mathbf{w})),
$$

which is where the Lie group structure appears (i.e. (7) states that the group multiplication function $\psi$ is associative).

Equation (6) is significant - it shows that crystals with uniform distributions of defects have a self similarity property determined by the dislocation density tensor (e.g. in the case where the dislocation density is zero, $\boldsymbol{\psi}$ may be chosen so that $\boldsymbol{\psi}(\mathbf{x}, \mathbf{u})=\mathbf{x}+\mathbf{u}$, so $\nabla_{1} \boldsymbol{\psi}(\mathbf{x}, \mathbf{u})=i d$, the identity tensor, and (6) implies that $\boldsymbol{\ell}_{a}(\mathbf{x}+\mathbf{u})=\boldsymbol{\ell}_{a}(\mathbf{x})$. Thus lattice vectors are constant, in this case). Therefore (6) is the generalization of the assumption, in the case of perfect crystals, that the lattice vector fields are translation invariant. Next, consider the basic assumption (in the case of perfect crystals) that one considers perfect lattices, a priori. This may be expressed as the assumption that one is to deal with sets of points obtained by flow through 'unit time' along the relevant lattice vector fields (since if $\boldsymbol{\ell}_{a}(\mathbf{x}) \equiv \mathbf{e}_{a}, a=1,2,3$, flow through 'unit time' starting at point $\mathbf{x}_{0}$, along vector field $\boldsymbol{\ell}_{a}(\cdot)$, requires that one solves $\dot{\mathbf{x}}(t)=\mathbf{e}_{a}, \mathbf{x}(0)=\mathbf{x}_{0}$, when $t$ is a real parameter and $\dot{\mathbf{x}}(t) \equiv \frac{d \mathbf{x}}{d t}(t)$. Thus flow along the three vector fields gives the three points $\mathbf{x}_{0}+\mathbf{e}_{1}, \mathbf{x}_{0}+\mathbf{e}_{2}, \mathbf{x}_{0}+\mathbf{e}_{3}$, and iterating this procedure backwards and forwards gives the set of points $\mathbf{x}_{0}+L$, when $L$ is the perfect lattice given by (1)). In the general case, when the crystal has non zero, uniform, dislocation density, I 
choose to adopt this last expression of this particular basic assumption, and note that it implies that one is dealing with structures other than perfect lattices, in general. In fact, the set of points produced in this way can be represented as a group, denote it $\tilde{G}$, with multiplication defined by the Lie group composition function $\psi \cdot \tilde{G}$ is generated by three elements $\mathbf{g}_{1}, \mathbf{g}_{2}, \mathbf{g}_{3}$, related to the flows along the three lattice vector fields, and $\tilde{G}$ has symmetries which correspond to different choices of the generators (in the perfect crystal case, $\boldsymbol{\psi}(\mathbf{x}, \mathbf{u})=\mathbf{x}+\mathbf{u}$, so the group 'multiplication' is addition, the group $\tilde{G}$ is the lattice $L$, the generators $\mathbf{g}_{1}, \mathbf{g}_{2}, \mathbf{g}_{3}$ are the basis vectors $\mathbf{e}_{1}, \mathbf{e}_{2}, \mathbf{e}_{3}$, and the symmetries of $\tilde{G}$ correspond to the different choices of basis vectors given by $(2))$. It turns out that symmetries of $\tilde{G}$ may be very simply expressed in a manner quite analogous to the requirement that $\gamma \in G L_{3}(\mathbb{Z})$, in the case of perfect crystals.

In general, when the dislocation density tensor (ddt.) is non zero, the corresponding lattice vector fields are not constant. The analogue of (3), in the general case, is the following; first one may choose right invariant lattice vector fields $\ell_{1}(\cdot), \ell_{2}(\cdot), \ell_{3}(\cdot)$ such that, if $F$ is a given linear transformation,

$$
F \ell_{a}(\mathbf{x})=\tilde{\gamma}_{a b} \ell_{b}(F \mathbf{x})
$$

where $\tilde{\gamma} \equiv\left(\tilde{\gamma}_{a b}\right)$ is a (constant) matrix. (I shall refer to these particular fields as Pontryagin's canonical fields). So, the point group of the crystal structure generated as above may be defined as follows: given the dislocation density, construct the set of points which represents the crystal structure, and construct the group $\tilde{G}$, with multiplication defined by the Lie group composition function $\boldsymbol{\psi}$. Determine the set of matrices $\tilde{\gamma}$ in $(8)$ which corresponds to the symmetries of $\tilde{G}$. Then the point group of the structure is the set of orthogonal transformations $F$ for which the corresponding matrices $\tilde{\gamma}$, in (8), are symmetries of $\tilde{G}$. Moreover, from (8) one obtains

$$
F=\tilde{\gamma}_{a b} \ell_{b}(\mathbf{0}) \otimes \mathbf{d}_{a}(\mathbf{0})
$$

where $\left\{\mathbf{d}_{1}(\cdot), \mathbf{d}_{2}(\cdot), \mathbf{d}_{3}(\cdot)\right\}$ is the set of fields which is dual to the set of vector fields, in the sense that

$$
\ell_{a}(\mathbf{x}) \cdot \mathbf{d}_{b}(\mathbf{x})=\delta_{a b},
$$

when $\delta_{a b}$ is the Kronecker delta, as before. Equation (9) is the analogue of (4).

Finally if one assumes that the energy density of a perfect crystal may be expressed as

$$
w=w\left(\left\{\mathbf{e}_{a}\right\}\right)
$$

where $\left\{\mathbf{e}_{a}\right\} \equiv\left\{\mathbf{e}_{1}, \mathbf{e}_{2}, \mathbf{e}_{3}\right\}$, then the assumption that the energy is independent of 'final orthogonal transformation (i.e. is 'objective') is

$$
w\left(\left\{\mathbf{R}_{a}\right\}\right)=w\left(\left\{\mathbf{e}_{a}\right\}\right),
$$

when $R$ is orthogonal. Let $C$ be the matrix with entries $\mathbf{e}_{a} \cdot \mathbf{e}_{b}$. Then (12) implies that $w$ may be written as

$$
w=\tilde{w}(C) .
$$

If one requires that $w$ just depends on the lattice $L$, then $w$ is independent of the choice of basis vectors $\left\{\mathbf{e}_{a}\right\}$ and so from (2), (13),

$$
\tilde{w}(C)=\tilde{w}\left(\gamma C \gamma^{T}\right), \quad \gamma \in G L_{3}(\mathbb{Z}) .
$$


Note that (14) is trivially satisfied if $\gamma \in \tilde{L}_{p}$, since $C=\gamma C \gamma^{T}$ in that case. I shall examine, in the sequel, how these calculations may be generalized when the energy density depends on the ddt. as well as the local values of the lattice vector fields.

The plan of the paper is the following: in the next section, I summarize basic ideas in the theory of Lie algebras/groups, so far as they appear to be necessary for the development outlined above; then I consider integrability properties of various (differential) systems which are involved in the passage from Lie algebra to Lie groups. Subsequently I use these ideas to give details of the calculations that are outlined above, and to calculate the point group of a defective crystal structure in a particularly simple case. Finally, I discuss the generalization of ideas in the last paragraph to defective crystals.

\section{Elements of Lie Theory}

\section{(i) Basics}

Let $G$ be a three dimensional Lie group, let $x \in G$ and let $e$ be the group identity element. Fix a system of coordinates in $\mathbb{R}^{3}$ and let the coordinates of the element $x$ be the components of a vector $\mathbf{x} \in U \subseteq \mathbb{R}^{3}$, with respect to a basis $\mathbf{e}_{i}, i=1,2,3$. Let $x \cdot y$ be the product of group elements $x, y$ and let $\psi: U \times U \rightarrow U$ be the composition function, such that the components of the vector $\psi(\mathbf{x}, \mathbf{y})$ are the coordinates of the group element $x \cdot y$, where $x, y$ correspond to vectors $\mathbf{x}, \mathbf{y}$, respectively. I prescribe that the system of coordinates is such that the coordinates of $e$ are components of the zero vector $\mathbf{0}$. Let $x^{-1}$ be the inverse of $x$, and let $\mathbf{x}^{-1}$ be the corresponding vector. Then

$$
\boldsymbol{\psi}(\mathbf{0}, \mathbf{x})=\boldsymbol{\psi}(\mathbf{x}, \mathbf{0})=\mathbf{x}, \quad \psi\left(\mathbf{x}^{-1}, \mathbf{x}\right)=\boldsymbol{\psi}\left(\mathbf{x}, \mathbf{x}^{-1}\right)=\mathbf{0} .
$$

Also the group product is associative, so

$$
\psi(\mathbf{x}, \boldsymbol{\psi}(\mathbf{y}, \mathbf{z}))=\psi(\psi(\mathbf{x}, \mathbf{y}), \mathbf{z}), \quad \mathbf{x}, \mathbf{y}, \mathbf{z} \in U .
$$

Let

$$
\left.A_{i j k} \equiv \frac{\partial^{2} \psi_{i}}{\partial x_{j} \partial y_{k}}(\mathbf{x}, \mathbf{y})\right|_{\mathbf{x}=\mathbf{y}=\mathbf{0}},
$$

when $\mathbf{x}=x_{i} \mathbf{e}_{i}$, etc., and let

$$
C_{i j k} \equiv A_{i j k}-A_{i k j}=\varepsilon_{s j k} \varepsilon_{s p q} A_{i p q},
$$

when $\varepsilon_{i j k}$ are the components of the permutation symbol. The constants $C_{i j k}$ are called the structure constants of the Lie algebra which corresponds to $G$, with respect to the given choice of coordinates. The associativity of the group product implies that the structure constants satisfy the Jacobi identity, namely that

$$
C_{i j k} C_{j r s}+C_{i j r} C_{j s k}+C_{i j s} C_{j k r}=0 .
$$

In general (that is, without reference to the above), a Lie algebra $\mathfrak{g}$ is a vector space together with a bilinear, skew-symmetric 'bracket' product $[\cdot, \cdot]: \mathfrak{g} \times \mathfrak{g} \rightarrow \mathfrak{g}$ which satisfies the identity,

$$
[\mathbf{X},[\mathbf{Y}, \mathbf{Z}]]+[\mathbf{Y},[\mathbf{X}, \mathbf{Z}]]+[\mathbf{Z},[\mathbf{X}, \mathbf{Y}]]=\mathbf{0}, \quad \mathbf{X}, \mathbf{Y}, \mathbf{Z} \in \mathfrak{g}
$$


Note that if the vector space is $\mathbb{R}^{3}$ and one sets

$$
[\mathbf{X}, \mathbf{Y}]=C_{i j k} X_{j} Y_{k} \mathbf{e}_{i}
$$

where the constants $C_{i j k}=-C_{i k j}$ are as above, then this particular bracket product is evidently bilinear and skew-symmetric, and it satisfies (20) by virtue of (19).

It is productive to introduce the notion of a right invariant vector field on $G$. If one fixes $y \in G$, then the function $\psi(\cdot, \mathbf{y}): U \rightarrow U$ represents 'multiplication on the right' by $y$, and the gradient of this function will be denoted $\nabla_{1} \boldsymbol{\psi}(\cdot, \mathbf{y})$. A vector field $\boldsymbol{\nu}$ is an object defined at each point $\mathbf{x} \in U \subseteq \mathbb{R}^{3}$ taking values in the tangent space (to the manifold $U$ ) at $\mathbf{x}$, thus $\boldsymbol{\nu}$ has components $\nu_{i}(\mathbf{x}), i=1,2,3$, and $\boldsymbol{\nu}=\nu_{i}(\mathbf{x}) \mathbf{e}_{i}$. The vector field $\nu$ is said to be right invariant with respect to the composition function $\psi$ if

$$
\boldsymbol{\nu}(\boldsymbol{\psi}(\mathbf{x}, \mathbf{y}))=\nabla_{1} \boldsymbol{\psi}(\mathbf{x}, \mathbf{y}) \boldsymbol{\nu}(\mathbf{x}), \quad \mathbf{x}, \mathbf{y} \in U .
$$

Given a vector field $\boldsymbol{\nu}$, the integral curve $\mathbf{x}(t)$ passing through initial point $\mathbf{x}_{0}$, associated with $\nu$, is the solution of the ordinary differential equation

$$
\frac{d \mathbf{x}}{d t}(t)=\boldsymbol{\nu}(\mathbf{x}(t)), \quad \mathbf{x}(0)=\mathbf{x}_{0} .
$$

It can be shown that the integral curve corresponding to a right invariant vector field is complete, in the sense that (23) has a solution well defined for all real values $t$. So, for each $t \in \mathbb{R}$, one can define a mapping $\exp (t \boldsymbol{\nu}): U \rightarrow U$ by

$$
\exp (t \boldsymbol{\nu})\left(\mathbf{x}_{0}\right)=\mathbf{x}(t), \quad \mathbf{x} \in U,
$$

when $\mathbf{x}(t)$ is the integral curve passing through initial point $\mathbf{x}_{0}$, associated with (the right invariant vector field) $\boldsymbol{\nu}$. One also defines the point $e^{t \boldsymbol{\nu}} \in U$ by

$$
e^{t \boldsymbol{\nu}} \equiv \exp (t \boldsymbol{\nu})(\mathbf{0})
$$

It is standard that

$$
\exp (t \boldsymbol{\nu})(\mathbf{x})=\boldsymbol{\psi}\left(e^{t \boldsymbol{\nu}}, \mathbf{x}\right)
$$

so that the flow corresponding to the mapping $\exp (t \boldsymbol{\nu})$ is simply related to the integral curve which passes through the origin. Indeed, notice that

$$
\begin{aligned}
\frac{d}{d t} \boldsymbol{\psi}\left(e^{t \boldsymbol{\nu}}, \mathbf{x}\right) & =\nabla_{1} \boldsymbol{\psi}\left(e^{t \boldsymbol{\nu}}, \mathbf{x}\right) \frac{d}{d t}\left(e^{t \boldsymbol{\nu}}\right) \\
& =\nabla_{1} \boldsymbol{\psi}\left(e^{t \boldsymbol{\nu}}, \mathbf{x}\right) \frac{d}{d t}[\exp (t \boldsymbol{\nu})(\mathbf{0})]=\nabla_{1} \boldsymbol{\psi}\left(e^{t \boldsymbol{\nu}}, \mathbf{x}\right) \frac{d \mathbf{x}}{d t}
\end{aligned}
$$

where $\mathbf{x}(t)$ satisfies $(23)$ with $\mathbf{x}(0)=\mathbf{0}$, so

$$
\frac{d}{d t} \boldsymbol{\psi}\left(e^{t \boldsymbol{\nu}}, \mathbf{x}\right)=\nabla_{1} \boldsymbol{\psi}\left(e^{t \boldsymbol{\nu}}, \mathbf{x}\right) \boldsymbol{\nu}(\mathbf{x})=\mathbf{x}\left(\boldsymbol{\psi}\left(e^{t \boldsymbol{\nu}}, \mathbf{x}\right)\right)
$$

by (22), since $\boldsymbol{\nu}$ is right invariant by hypothesis. Hence $\boldsymbol{\psi}\left(e^{t \boldsymbol{\nu}}, \mathbf{x}\right)$ is the unique solution of (23), with initial condition $\mathbf{x}(0)=\boldsymbol{\psi}\left(e^{0 \boldsymbol{\nu}}, \mathbf{x}\right)=\boldsymbol{\psi}(0, \mathbf{x})=\mathbf{x}$. This proves (26).

The points $\{\mathbf{x}(t), t \in \mathbb{R}\}$ which make up the integral curve of a right invariant field $\boldsymbol{\nu}$ are the elements of a one parameter subgroup of $G$, so that

$$
\boldsymbol{\psi}(\mathbf{x}(t), \mathbf{x}(s))=\mathbf{x}(t+s), \quad t, s \in \mathbb{R},
$$


and the converse is also true (see Pontryagin [2], for example).

Right invariant vector fields exist, and the set of right invariant vector fields is a vector space with respect to pointwise addition and (real) scalar multiplication. For by differentiating (16) with respect to $\mathbf{x}$ and putting $\mathbf{x}=\mathbf{0}$,

$$
\nabla_{1} \psi(\mathbf{0}, \boldsymbol{\psi}(\mathbf{y}, \mathbf{z}))=\nabla_{1} \psi(\mathbf{y}, \mathbf{z}) \nabla_{1} \psi(\mathbf{0}, \mathbf{y})
$$

Here, let $\mathbf{e}_{1}, \mathbf{e}_{2}, \mathbf{e}_{3}$ be an orthonormal basis of $\mathbb{R}^{3}$ and define

$$
\boldsymbol{\ell}_{a}(\mathbf{x})=\nabla_{1} \boldsymbol{\psi}(\mathbf{0}, \mathbf{x}) \mathbf{e}_{a}, \quad a=1,2,3 .
$$

Thus (30) gives

$$
\ell_{a}(\boldsymbol{\psi}(\mathbf{y}, \mathbf{z}))=\nabla_{1} \psi(\mathbf{y}, \mathbf{z}) \ell_{a}(\mathbf{y})
$$

so that the vector fields which are explicitly defined by (31) are right invariant. Moreover these particular vector fields form a basis for the vector space of right invariant fields: if $\boldsymbol{\nu}$ is an arbitrary right invariant field, then from (22)

$$
\begin{aligned}
\boldsymbol{\nu}(\mathbf{x}) & =\nabla_{1} \boldsymbol{\psi}(\mathbf{0}, \mathbf{x}) \boldsymbol{\nu}(\mathbf{0}) \\
& =\left(\nabla_{1} \boldsymbol{\psi}(\mathbf{0}, \mathbf{x}) \mathbf{e}_{a}\right)\left(\mathbf{e}_{a} \cdot \boldsymbol{\nu}(\mathbf{0})\right) \\
& =\left(\mathbf{e}_{a} \cdot \boldsymbol{\nu}(\mathbf{0})\right) \boldsymbol{\ell}_{a}(\mathbf{x}),
\end{aligned}
$$

via (31). Further, each right invariant field is determined by its value at the origin (once $\boldsymbol{\psi}$, and so $\boldsymbol{\ell}_{a}$, is known).

\section{(ii) Lie bracket}

Let $\boldsymbol{\nu}, \boldsymbol{\omega}$ be two right invariant vector fields. Let $\boldsymbol{\theta}(t, \mathbf{x})$ be the vector corresponding to the group element $e^{-\sqrt{t} \boldsymbol{\omega}} \cdot e^{-\sqrt{t} \boldsymbol{\nu}} \cdot e^{\sqrt{t} \boldsymbol{\omega}} \cdot e^{\sqrt{t} \boldsymbol{\nu}} \cdot x$ where $a \cdot b$ is the group element corresponding to $\boldsymbol{\psi}(\mathbf{a}, \mathbf{b}), a, b \in U$ (and $e^{\sqrt{t} \boldsymbol{\nu}}$, for example, in the above expression, is understood to be the group element corresponding to the point $e^{\sqrt{t} \boldsymbol{\nu}} \in U$ defined by an analogue of (25)). Then according to Olver [3],

$$
\lim _{t \rightarrow 0+} \frac{\boldsymbol{\theta}(t, \mathbf{x})-\boldsymbol{\theta}(0, \mathbf{x})}{t}=[\boldsymbol{\nu}, \boldsymbol{\omega}](\mathbf{x}),
$$

where the vector field $[\boldsymbol{\nu}, \boldsymbol{\omega}](\cdot)$ is called the Lie bracket of $\boldsymbol{\nu}, \boldsymbol{\omega}$, and is defined by

$$
[\boldsymbol{\nu}, \boldsymbol{\omega}](\mathbf{x})=((\boldsymbol{\nu} \cdot \nabla) \boldsymbol{\omega}-(\boldsymbol{\omega} \cdot \nabla) \boldsymbol{\nu})(\mathbf{x}) .
$$

The Lie bracket of right invariant vector fields is itself a right invariant vector field, and as such it is determined by its value at the origin (given the form of the composition function $\psi$ ). So, given right invariant fields $\mathbf{x}, \mathbf{y}$ with $\mathbf{x}(\mathbf{0})=\mathbf{X}, \mathbf{y}(\mathbf{0})=\mathbf{Y}$, define

$$
[\mathbf{X}, \mathbf{Y}]=[\mathbf{x}, \mathbf{y}](0), \quad \mathbf{X}, \mathbf{Y} \in \mathbb{R}^{3} .
$$

This is a skew-symmetric, bilinear bracket product on $\mathbb{R}^{3} \times \mathbb{R}^{3}$, it satisfies the identity (20), and so defines a Lie algebra on $\mathbb{R}^{3}$. In this way one may regard the set of right invariant vector fields on the Lie group $G$ as a Lie algebra on $\mathbb{R}^{3}$. Further one may verify (using the definition of $\boldsymbol{\theta}(t, \mathbf{x})$, above) that (36) implies that (21) holds, with $C_{i j k}$ defined by (18). 
(iii) Algebra and group homomorphisms

A linear transformation $L: \mathfrak{g} \rightarrow \mathfrak{h}$ is a Lie algebra homomorphism if it preserves the Lie bracket operation in the sense that

$$
[L \mathbf{X}, L \mathbf{Y}]_{\mathfrak{h}}=L[\mathbf{X}, \mathbf{Y}]_{\mathfrak{g}}, \quad \mathbf{X}, \mathbf{Y} \in \mathfrak{g}
$$

where $[\cdot, \cdot]_{\mathfrak{g}},[\cdot, \cdot]_{\mathfrak{h}}$ denotes the bracket products in $\mathfrak{g}$ and $\mathfrak{h}$, respectively. Assume that $\mathfrak{g}=\mathfrak{h}=\mathbb{R}^{3}$, and express the components of $L \mathbf{X}, \mathbf{X}$ with respect to the same basis. Then if $L \mathbf{X}$ has components $L_{i j} X_{j}$, etc., and the structure constants of $\mathfrak{g}, \mathfrak{h}$ are denoted $C_{i j k}^{\mathfrak{g}}, C_{i j k}^{\mathfrak{h}}$, one obtains from (37)

$$
C_{i j k}^{\mathfrak{h}} L_{j p} L_{h q} X_{p} Y_{q}=L_{i r} C_{r p q}^{\mathfrak{g}} X_{p} Y_{q}
$$

so that

$$
C_{i j k}^{\mathfrak{h}} L_{j p} L_{k q}=L_{i r} C_{r p q}^{\mathfrak{g}}
$$

since $X_{p}, Y_{q}$ are arbitrary.

Now suppose that $\mathfrak{g}$ is the Lie algebra of a simply connected Lie group $\mathfrak{G}$, that $\mathfrak{h}$ is the Lie algebra of a Lie group $\mathfrak{H}$, and that $L$ is as above. Suppose that these are three dimensional Lie groups, so that the coordinates of the respective group elements are elements of sets $U_{\mathbf{g}}, U_{\mathbf{h}} \subseteq \mathbb{R}^{3}$. Then there exists a unique Lie group homomorphism, whose expression in coordinates is $\varphi: U_{\mathbf{g}} \rightarrow U_{\mathbf{h}}$, such that

$$
\nabla \varphi(\mathbf{0})=L
$$

The fact that $\varphi$ is a Lie group homomorphism means that

$$
\psi_{\mathbf{h}}(\varphi(\mathbf{x}), \varphi(\mathbf{y}))=\varphi\left(\psi_{\mathbf{g}}(\mathbf{x}, \mathbf{y})\right), \mathbf{x}, \mathbf{y} \in U_{\mathbf{g}}
$$

where $\psi_{\mathbf{g}}, \psi_{\mathbf{h}}$ are representations of the group composition in $U_{\mathbf{g}}, U_{\mathbf{h}}$, respectively.

It is straightforward to show that, if (41) holds, and $\nabla \varphi(\mathbf{0})=L$, then so does (37). For by differentiating (41) successively with respect to $\mathbf{x}, \mathbf{y}$ and putting $\mathbf{x}=\mathbf{y}=\mathbf{0}$, noting that $\varphi(\mathbf{0})=\mathbf{0}$,

$\left(\nabla_{12} \boldsymbol{\psi}_{\mathbf{h}}\right)_{i, k r}(\mathbf{0}, \mathbf{0}) \varphi_{k, p}(\mathbf{0}) \varphi_{r, q}(\mathbf{0})=\varphi_{i, j q}(\mathbf{0})\left(\nabla_{1} \boldsymbol{\psi}_{\mathfrak{g}}\right)_{j, p}(\mathbf{0}, \mathbf{0})+\varphi_{i, j}(\mathbf{0})\left(\nabla_{12} \boldsymbol{\psi}_{\mathfrak{g}}\right)_{j, p q}(\mathbf{0}, \mathbf{0})$

But $\boldsymbol{\psi}_{\mathfrak{g}}(\mathbf{x}, \mathbf{0})=\mathbf{x}$ so $\left(\nabla_{1} \boldsymbol{\psi}_{\mathbf{g}}\right)_{i, k}(\mathbf{0}, \mathbf{0})=\delta_{i k}$. So noting (18), and putting $\varphi_{i, j}(\mathbf{0})=L_{i j}$, (42) gives (38) by antisymmetrization with respect to $p, q$. On the other hand, the proof that (37) implies the existence of a unique corresponding Lie group homomorphism is a major result of Lie theory, and I refer to Varadarajan [4], Warner [5] for the details.

I note that there is a simple relation between the structure constants of a Lie group and the dislocation density tensor of a uniformly defective crystal, so that equation (39) may be reexpressed as a constraint on the dislocation density. This fact will be useful later on. 
(iv) Integrability conditions

(a) The basis right invariant fields $\ell_{1}(\cdot), \ell_{2}(\cdot), \ell_{3}(\cdot)$ defined by (31), with $\mathbf{e}_{a}$ orthonormal, satisfy

$$
\boldsymbol{\ell}_{a}(\boldsymbol{\psi}(\mathbf{x}, \mathbf{y}))=\nabla_{1} \boldsymbol{\psi}(\mathbf{x}, \mathbf{y}) \boldsymbol{\ell}_{a}(\mathbf{x}), \quad a=1,2,3, \boldsymbol{\ell}_{a}(\mathbf{0})=\mathbf{e}_{a}
$$

Let $\mathbf{d}_{a}(\cdot), a=1,2,3$, be dual to the basis right invariant fields, so that

$$
\ell_{a}(\mathbf{x}) \cdot \mathbf{d}_{b}(\mathbf{x})=\delta_{a b}, \quad a=1,2,3, \mathbf{x} \in U
$$

Then

$$
\nabla_{1}^{T} \boldsymbol{\psi}(\mathbf{x}, \mathbf{y}) \mathbf{d}_{a}(\boldsymbol{\psi}(\mathbf{x}, \mathbf{y}))=\mathbf{d}_{a}(\mathbf{x}), \quad a=1,2,3, \mathbf{d}_{a}(\mathbf{0})=\mathbf{e}_{a},
$$

where $\nabla_{1}^{T} \boldsymbol{\psi}$ denotes the transpose of $\nabla_{1} \boldsymbol{\psi}$. Note that the dual (or co-vector) fields $\mathbf{d}_{1}(\cdot), \mathbf{d}_{2}(\cdot), \mathbf{d}_{3}(\cdot)$ have quite different transformational properties to the vector fields $\ell_{1}(\cdot), \ell_{2}(\cdot), \ell_{3}(\cdot)$. (This distinction is often emphasized by using upper indices to label dual vector fields, but I choose not to do so - here the kernel letter, $\ell$ or $\mathbf{d}$, indicates whether or not the object is a vector or a co-vector).

The condition on the fields $\mathbf{d}_{a}(\cdot)$ which guarantees that equation (45) has a solution for $\boldsymbol{\psi}$, satisfying $\boldsymbol{\psi}(\mathbf{0}, \mathbf{x})=\boldsymbol{\psi}(\mathbf{x}, \mathbf{0})=\mathbf{x}$, is that

$$
d_{a j, i}(\mathbf{x})-d_{a i, j}(\mathbf{x})=\gamma_{a r s} d_{r i}(\mathbf{x}) d_{s j}(\mathbf{x})
$$

for some constants $\gamma_{\text {ars }}$. Moreover, if (46) holds the function $\psi$ is associative, and the solution $\boldsymbol{\psi}$ is unique. Of course, if (43) is given, then (45) does indeed have a solution $\psi$, and the constants $\gamma_{\text {ars }}$ satisfy

$$
\begin{aligned}
\gamma_{a i j} & =d_{a j, i}(\mathbf{0})-d_{a i, j}(\mathbf{0})=-\ell_{j a, i}(\mathbf{0})+\ell_{i a, j}(\mathbf{0}) \\
& =-\frac{\partial^{2} \psi_{a}}{\partial x_{j} \partial y_{i}}(\mathbf{0}, \mathbf{0})+\frac{\partial^{2} \psi_{a}}{\partial x_{i} \partial y_{j}}(\mathbf{0}, \mathbf{0})=C_{a i j},
\end{aligned}
$$

via (43), (44) and (18).

That is to say, if fields $\ell_{a}(\cdot)$ are given, they may be considered to be right invariant fields on a Lie group with composition function $\psi$ if the quantities $\gamma_{\text {ars }}$ defined by (46) are constant. In that case, those constants are the structure constants of the corresponding Lie algebra, and it turns out that the quantities $\gamma_{\text {ars }}$ are constant if and only if the dislocation density tensor (which is defined directly in terms of $\left.\mathbf{d}_{a}(\cdot)\right)$ is constant - in fact, from (46), (44),

$$
\left(\nabla \wedge \mathbf{d}_{a}\right)_{k}=\varepsilon_{k i j} d_{a j, i}=\frac{1}{2} \varepsilon_{k i j}\left(d_{a j, i}-d_{a i, j}\right)=\frac{1}{2} \gamma_{a r s} \varepsilon_{k i j} d_{r i} d_{s j} .
$$

So putting

$$
n(\mathbf{x}) \equiv \mathbf{d}_{1}(x) \cdot \mathbf{d}_{2}(\mathbf{x}) \wedge \mathbf{d}_{3}(\mathbf{x}),
$$

one obtains from (47) that

$$
\begin{aligned}
\nabla \wedge \mathbf{d}_{a} \cdot \mathbf{d}_{q} & =\frac{1}{2} \gamma_{a r s} \varepsilon_{k i j} d_{r i} d_{s j} d_{q k} \\
& =\frac{1}{2} \gamma_{a r s} \varepsilon_{r s q} n .
\end{aligned}
$$


Define the dislocation density tensor $\mathcal{S}$ by specifying its components $S_{a b}$ :

$$
\mathcal{S}_{a b} \equiv \frac{\nabla \wedge \mathbf{d}_{a} \cdot \mathbf{d}_{b}}{n} .
$$

Then from (50)

$$
\mathcal{S}_{a q}=\frac{1}{2} \gamma_{a r s} \varepsilon_{r s q}
$$

So, in the case that the quantities $\gamma_{\text {ars }}$ are constant, so is the dislocation density $\mathcal{S}$. Then the quantities $\gamma_{a r s}=C_{a r s}=-C_{a s r}$, and from (52), $C_{a i j}=\gamma_{a i j}=\varepsilon_{i j k} \mathcal{S}_{a k}$. For arbitrary right invariant fields, the connection between structure constants and dislocation density is

$$
C_{i j k} \ell_{r j}(\mathbf{0}) \ell_{s k}(\mathbf{0})=\varepsilon_{p r s} S_{k p} \ell_{k i}(\mathbf{0}),
$$

see Elzanowski and Parry [10] for a derivation. Thus the constraint that one considers fields with uniform dislocation density entails that the quantities $\gamma_{a i j}$, above, are constant, so it implies that equation (43) has a solution for the composition function $\boldsymbol{\psi}$, and thus the entire apparatus of Lie group theory becomes available for the purpose of studying defects in crystal. Remarks to this effect may be found in Belinfante [6], Bilby [7], but the observation has not been developed since in any way, to my knowledge (other than in Parry [8], Cermelli and Parry [9], Elzanowski and Parry [10]).

(b) In elasticity theory, a reference line element $\mathbf{L}$ is deformed, by elastic deformation $\boldsymbol{\theta}$, to current line element $\boldsymbol{\ell}$ defined by

$$
\ell=\nabla \boldsymbol{\theta}(\mathbf{x}) \mathbf{L} .
$$

So, for a crystal with defects, with texture defined by lattice vector fields $\boldsymbol{\ell}_{a}(\cdot)$, it is natural to require that an elastic deformation $\boldsymbol{\theta}$ of the crystal deforms the fields $\ell_{a}(\cdot)$ to fields $\ell_{a}^{\prime}(\cdot)$ defined by

$$
\ell_{a}^{\prime}(\boldsymbol{\theta}(\mathbf{x}))=\nabla \boldsymbol{\theta}(\mathbf{x}) \ell_{a}(x) .
$$

One asks, if fields $\boldsymbol{\ell}_{a}^{\prime}(\cdot)$ and $\boldsymbol{\ell}_{a}(\cdot)$ are given, what is the condition that (55) has a solution for the deformation $\boldsymbol{\theta}$ ? According to Caratheodory [11], Olver [3], Parry [8], the condition is that the dislocation density corresponding to fields $\ell_{a}^{\prime}(\cdot)$ is constant and equals the dislocation density corresponding to fields $\ell_{a}(\cdot)$ (one has to require that (55) has a solution for arbitrary $\mathbf{x}_{0}, \mathbf{y}_{0}$, with $\mathbf{y}_{0}=\boldsymbol{\theta}\left(\mathbf{x}_{0}\right)$, in order to derive this result). According to part (a) above, it follows that (43) has a solution in the two cases where the given fields are $\boldsymbol{\ell}_{a}(\cdot), \ell_{a}^{\prime}(\cdot)$. Thus in (55), the fields $\boldsymbol{\ell}_{a}(\cdot)$ are right invariant fields for some Lie group $G$, the fields $\ell_{a}^{\prime}(\cdot)$ are right invariant fields for some Lie group $G^{\prime}$, with coordinates in corresponding sets $U, U^{\prime}$. The deformation $\boldsymbol{\theta}$, in this case, expresses a mapping between different Lie groups. In fact if (43) holds and one defines (associative) $\psi^{\prime}$ by

$$
\boldsymbol{\psi}^{\prime}(\boldsymbol{\theta}(\mathbf{x}), \boldsymbol{\theta}(\mathbf{y}))=\boldsymbol{\theta}(\boldsymbol{\psi}(\mathbf{x}, \mathbf{y}))
$$

$\ell_{a}^{\prime}(\cdot)$ by $(55)$, then

$$
\ell_{a}^{\prime}\left(\boldsymbol{\psi}^{\prime}\left(\mathbf{x}^{\prime}, \mathbf{y}^{\prime}\right)\right)=\nabla_{1} \boldsymbol{\psi}^{\prime}\left(\mathbf{x}^{\prime}, \mathbf{y}^{\prime}\right) \ell_{a}^{\prime}\left(\mathbf{x}^{\prime}\right), \quad a=1,2,3,
$$

which shows that $\ell_{a}^{\prime}(\cdot)$ is right invariant with respect to $\boldsymbol{\psi}^{\prime}$, and $\boldsymbol{\theta}$ is a Lie group homomorphism (by comparison of (41) and (56)). 


\section{(v) Correspondence between Lie algebras and Lie groups}

An isomorphism of Lie algebras/Lie groups is a Lie algebra/Lie group homomorphism which is one to one. According to Varadarajan [4], Warner [5], there is a one to one correspondence between isomorphism classes of Lie algebras and isomorphism classes of Lie groups.

A linear transformation of vector spaces can be regarded as a change of basis, and according to the previous section an isomorphism class of Lie groups corresponds to those groups which are obtained from a given group by elastic deformation. Therefore, in order to make the correspondence which is stated above explicit, it is sufficient to illustrate how one may construct from a given set of structure constants (simplified by change of basis, if required), just one Lie group with particular properties. The set of all Lie groups with given structure constants (to within change of basis) is then obtained by elastic deformation of that one group. In the following section, I recall how Pontryagin [2] constructs a 'canonical' set of right invariant fields, given structure constants. The right invariant fields so constructed satisfy $\boldsymbol{\ell}_{a}(\mathbf{0})=\mathbf{e}_{a}$, and the group composition function may then be obtained by solution of (43).

\section{(vi) Pontryagin's canonical fields}

Pontryagin constructs basis right invariant fields such that the set of points determined by the flow of these fields (through the origin) is a straight line. He shows that such fields $\ell_{a}(\cdot)$ may be determined by solving the ordinary differential system

$$
\dot{\omega}_{i j}=\delta_{i j}+C_{i p q} x_{p} \omega_{q j}, \quad \omega_{i j}(0)=0,
$$

for functions $\omega_{i j}(t)$ (where $\dot{\omega}_{i j}=\frac{d \omega_{i j}}{d t}(t)$ ), and putting

$$
d_{i j}(\mathbf{x})=\omega_{i j}(1),
$$

noting that $\mathbf{x}$ appears as a parameter in (58). Parry [8] shows that this procedure yields the basic dual fields

$$
\mathbf{d}_{a}(\mathbf{x})=\left(\frac{e^{A}-\mathbb{I}}{A}\right)^{T} \mathbf{e}_{a}
$$

where

$$
A \equiv\left(C_{i p q} x_{p}\right)
$$

and the symbol $\frac{e^{A}-\mathbb{I}}{A}$ is defined by

$$
\frac{e^{A}-\mathbb{I}}{A}=\mathbb{I}+\frac{A}{2 !}+\frac{A^{2}}{3 !}+\frac{A^{3}}{4 !}+\ldots
$$

Let $C_{i j k}^{\prime}, x_{i}^{\prime}$ be defined by

$$
C_{i j k}^{\prime} \tilde{L}_{j p} \tilde{L}_{k q}=\tilde{L}_{i r} C_{r p q}, \quad x_{i}^{\prime}=\tilde{L}_{i p} x_{p} .
$$

Then

$$
A^{\prime} \equiv\left(C_{i p q}^{\prime} x_{p}^{\prime}\right)
$$


is such that

$$
A^{\prime}=\tilde{L} A \tilde{L}^{-1} .
$$

Now recognize that the basis dual fields in (60) depend on the structure constants as well as the variable $\mathbf{x}$, and acknowledge this fact by writing

$$
\mathbf{d}_{a}(\mathbf{x}) \equiv \mathbf{d}_{a}(C, \mathbf{x}) .
$$

Then (60) is rewritten as

$$
\mathbf{d}_{a}(C, \mathbf{x})=\left(\mathbb{I}+\frac{A}{2 !}+\frac{A^{2}}{3 !}+\ldots\right)^{T} \mathbf{e}_{a}
$$

so

$$
\begin{aligned}
\mathbf{d}_{a}\left(C^{\prime}, \mathbf{x}^{\prime}\right) & =\left(\mathbb{I}+\frac{A^{\prime}}{2 !}+\frac{A^{\prime} 2}{3 !}+\ldots\right)^{T} \mathbf{e}_{a} \\
& =\tilde{L}^{-T}\left(\mathbb{I}+\frac{A}{2 !}+\frac{A^{2}}{3 !}+\ldots\right)^{T} \tilde{L}^{T} \mathbf{e}_{a}
\end{aligned}
$$

Thus

$$
\tilde{L}^{T} \mathbf{d}_{a}\left(C^{\prime}, \mathbf{x}^{\prime}\right)=\mathbf{d}_{b}(C, \mathbf{x})\left(\mathbf{e}_{b} \cdot \tilde{L}^{T} \mathbf{e}_{a}\right)
$$

or

$$
\tilde{L}_{j i} d_{a j}\left(C^{\prime}, \mathbf{x}^{\prime}\right)=d_{b i}(C, \mathbf{x}) \tilde{L}_{a b}
$$

This implies

$$
\tilde{L} \ell_{a}(C, \mathbf{x})=\tilde{L}_{b a} \ell_{b}\left(C^{\prime}, \tilde{L} \mathbf{x}\right)
$$

which is the precise expression of (8) in the case that $\boldsymbol{\ell}_{b}(\mathbf{0})=\mathbf{e}_{b}$. (If $\boldsymbol{\ell}_{a}(\mathbf{0})=\mathbf{e}_{a}$, (8) gives $\tilde{\gamma}^{T}=F$. Put $\tilde{L}=F$ in (71) and compare). Note that (71) holds identically for all nonsingular $\tilde{L}$.

Suppose now that $\tilde{\ell}_{a}(\cdot)$ is an arbitrary choice of three right invariant fields, in the same coordinate system. These fields may be expressed in terms of the basic fields $\boldsymbol{\ell}_{a}(\cdot)$ defined as above:

$$
\tilde{\ell}_{a}(\cdot)=\nu_{a b} \ell_{b}(\cdot)
$$

according to (33). Then (71) gives

$$
\begin{aligned}
\tilde{L} \tilde{\boldsymbol{\ell}}_{c}(C, \mathbf{x}) & =\tilde{L}\left(\nu_{c b} \boldsymbol{\ell}_{b}(C, \mathbf{x})\right)=\nu_{c b}\left(\tilde{L} \boldsymbol{\ell}_{b}(C, \mathbf{x})\right) \\
& =\nu_{c b} \tilde{L}_{a b} \boldsymbol{\ell}_{b}\left(C^{\prime}, \tilde{L} \mathbf{x}\right) \\
& =\nu_{c b} \tilde{L}_{a b} \nu_{b d}^{-1} \tilde{\boldsymbol{\ell}}_{d}\left(C^{\prime}, \tilde{L} \mathbf{x}\right) .
\end{aligned}
$$

Thus

$$
\tilde{L} \tilde{\ell}_{c}(C, \mathbf{x})=\tilde{\gamma}_{c d} \tilde{\ell}_{d}\left(C^{\prime}, \tilde{L} \mathbf{x}\right)
$$

where

$$
\tilde{\gamma}=\nu \tilde{L}^{T} \nu^{-1}
$$

and $\nu$ is the matrix with elements $\left(\nu_{a b}\right)$. Equation (74) is the required version of (8), appropriate to arbitrary right invariant fields $\tilde{\ell}_{b}(\cdot)$, in Pontryagin's canonical coordinate system, where the flows along the right invariant fields (through the origin) are straight lines. 


\section{(vii) Representation of arbitrary right invariant fields}

The duals of Pontryagin's basic canonical fields are defined via (59), and the fields themselves satisfy (71). If $\boldsymbol{\theta}$ is an elastic deformation (or group homomorphism), these basic canonical fields $\ell_{a}(\cdot)$ transform to right invariant fields $\ell_{a}^{\prime}(\cdot)$ defined by

$$
\ell_{a}^{\prime}(\boldsymbol{\theta}(\mathbf{x}))=\nabla \boldsymbol{\theta}(\mathbf{x}) \ell_{a}(\mathbf{x})
$$

so that

$$
\ell_{a}^{\prime}(\mathbf{y})=\nabla \boldsymbol{\theta}\left(\boldsymbol{\theta}^{-1}(\mathbf{y})\right) \ell_{a}\left(\boldsymbol{\theta}^{-1}(\mathbf{y})\right)
$$

An arbitrary right invariant field (in the isomorphism class of the given Lie group) therefore has the form

$$
\tilde{\ell}_{a}(\mathbf{y})=\gamma_{a b} \nabla \boldsymbol{\theta}\left(\boldsymbol{\theta}^{-1}(\mathbf{y})\right) \boldsymbol{\ell}_{b}\left(\boldsymbol{\theta}^{-1}(\mathbf{y})\right),
$$

for some matrix $\gamma \equiv\left(\gamma_{a b}\right)$, some elastic deformation $\boldsymbol{\theta}$. I show here that the mapping $\boldsymbol{\theta}$, in $(78)$, may be chosen so that $\nabla \boldsymbol{\theta}(\mathbf{0})=$ id., and that as a consequence the representation (78) is unique modulo elastic 'self-symmetries' of the fields $\boldsymbol{\ell}_{a}(\cdot)$.

To do this, note that from $(71)$

$$
\ell_{a}(C, \mathbf{x})=\tilde{L}^{-1} \ell_{b}\left(C^{\prime}, \tilde{L} \mathbf{x}\right) \tilde{L}_{b a}
$$

so that (78) may be written as

$$
\begin{aligned}
\tilde{\boldsymbol{\ell}}_{a}(\mathbf{y}) & =\gamma_{a b} \nabla \boldsymbol{\theta}\left(\boldsymbol{\theta}^{-1}(\mathbf{y})\right)\left[\tilde{L}^{-1} \ell_{c}\left(C^{\prime}, \tilde{L} \boldsymbol{\theta}^{-1}(\mathbf{y})\right) \tilde{L}_{c b}\right] \\
& =\left(\gamma_{a b} \tilde{L}_{c b}\right)\left[\nabla \boldsymbol{\varphi}^{-1}(\mathbf{y})\right]^{-1} \ell_{c}\left(C^{\prime}, \tilde{L} \boldsymbol{\theta}^{-1}(\mathbf{y})\right)
\end{aligned}
$$

where I have noted that

$$
\nabla \boldsymbol{\theta}\left(\boldsymbol{\theta}^{-1}(\mathbf{y})\right)=\left[\nabla \boldsymbol{\theta}^{-1}(\mathbf{y})\right]^{-1}
$$

and also put $\varphi^{-1}(\mathbf{y})=\tilde{L} \boldsymbol{\theta}^{-1}(\mathbf{y})$, so that if $\mathbf{y}=\boldsymbol{\theta}(\mathbf{x}), \mathbf{x}=\boldsymbol{\theta}^{-1}(\mathbf{y})$, then $\varphi^{-1}(\boldsymbol{\theta}(\mathbf{x}))=$ $\tilde{L} \mathbf{x}$ so

$$
\nabla \varphi^{-1}(\mathbf{y}) \nabla \boldsymbol{\theta}(\mathbf{x})=\tilde{L}
$$

Therefore

$$
\tilde{\ell}_{a}(\mathbf{y})=\left(\gamma_{a b} \tilde{L}_{c b}\right) \nabla \varphi\left(\varphi^{-1}(\mathbf{y})\right) \boldsymbol{\ell}_{c}\left(C^{\prime}, \varphi^{-1}(\mathbf{y})\right)
$$

using an identity analogous to (81). Equation (83) is in a form analogous to (78), where the arbitrary function $\boldsymbol{\theta}$ is replaced by $\boldsymbol{\varphi}$, with $\varphi^{-1}(\mathbf{y})=\tilde{L} \boldsymbol{\theta}^{-1}(\mathbf{y})$, so $\boldsymbol{\varphi}(\mathbf{x})=\boldsymbol{\theta}\left(\tilde{L}^{-1} \mathbf{x}\right)$.

Thus $\nabla \boldsymbol{\varphi}(\mathbf{0})=\nabla \boldsymbol{\theta}(\mathbf{0}) \tilde{L}^{-1}$, and since $\tilde{L}$ is arbitrary one may assume that $\nabla \boldsymbol{\varphi}(\mathbf{0})=$ id. in (83) (and so also that $\nabla \boldsymbol{\theta}(\mathbf{0})=$ id. in (78)). Note that the basis vector fields in (83) correspond to structure constants $C^{\prime}$, whereas (tacitly) the basis fields in (78) depend on structure constants $C$ - the point is that arbitrary right invariant fields (in the isomorphism class of the given Lie group) may be represented in the form (78), with $\nabla \boldsymbol{\theta}(\mathbf{0})=$ id., for some choice of structure constants (in the isomorphism class of the corresponding Lie algebra). 
Now suppose that a given set of right invariant fields has two distinct representations of the form (78), so that

$$
\gamma_{a b} \nabla \boldsymbol{\theta}\left(\boldsymbol{\theta}^{-1}(\mathbf{y})\right) \boldsymbol{\ell}_{b}\left(C, \boldsymbol{\theta}^{-1}(\mathbf{y})\right)=\bar{\gamma}_{a b} \nabla \overline{\boldsymbol{\theta}}\left(\overline{\boldsymbol{\theta}}^{-1}(\mathbf{y})\right) \boldsymbol{\ell}_{b}\left(C, \bar{\theta}^{-1}(\mathbf{y})\right)
$$

with

$$
\nabla \boldsymbol{\theta}(\mathbf{0})=\nabla \overline{\boldsymbol{\theta}}(\mathbf{0})=\text { id., } \boldsymbol{\theta}(\mathbf{0})=\overline{\boldsymbol{\theta}}(\mathbf{0})=\mathbf{0} .
$$

Putting $\mathbf{y}=\mathbf{0}$ in (79), recalling that $\boldsymbol{\ell}_{b}(C, \mathbf{0})=\mathbf{e}_{b}$, one obtains that $\gamma_{a b}=\bar{\gamma}_{a b}$. This implies that (84) may be expressed in the form:

$$
\boldsymbol{\ell}_{a}(C, \mathbf{p}(x))=\nabla \mathbf{p}(\mathbf{x}) \boldsymbol{\ell}_{a}(C, \mathbf{x}), \quad \text { where } \quad \mathbf{p} \equiv \boldsymbol{\theta}^{-1} \cdot \overline{\boldsymbol{\theta}}
$$

so that $\mathbf{p}$ is an elastic self-symmetry of the fields $\boldsymbol{\ell}_{a}(\cdot)$. So if $\bar{\gamma}_{a b}, \overline{\boldsymbol{\theta}}$ provide any other representation of the form (78), then $\bar{\gamma}_{a b}=\gamma_{a b}, \overline{\boldsymbol{\theta}}=\boldsymbol{\theta} \cdot \mathbf{p}$.

\section{Point group symmetries of defective crystal structures}

According to the assumption outlined in the introduction, I consider sets of points obtained by forward and backward iteration of the flow through 'unit time' along three right invariant vector fields. This is the generalization, to the case of crystals with uniform distributions of defects, of the idea that perfect crystals are represented by sets of points which are lattices, in the mathematical sense. Starting at a given point (respectively, group element) denoted by $\mathbf{x}$ (resp. $x$ ), flow through unit time along the lattice vector field $\ell_{a}(\cdot)$ produces the point (resp. group element) denoted $\psi\left(e^{\ell_{a}}, \mathbf{x}\right)$ (resp. $\left.e^{\ell_{a}} \cdot x\right)$, according to (26).

Correspondingly, flow through time -1 (i.e. 'backwards') along the same vector field produces the point (resp. group element) denoted $\boldsymbol{\psi}\left(e^{-\boldsymbol{\ell}_{a}}, \mathbf{x}\right)$ (resp. $\left.e^{-\boldsymbol{\ell}_{a}} \cdot x\right)$. Thus the iteration procedure produces the set of points which correspond to group elements

$$
\alpha_{1} \cdot \alpha_{2} \cdot \alpha_{3} \cdots \cdot \alpha_{n} \cdot x
$$

where $n$ is arbitrary, and where each $\alpha_{i}, i=1, \ldots n$ is one of the group elements $e^{\ell_{1}}$, $e^{\ell_{2}}, e^{\ell_{3}}, e^{-\ell_{1}}, e^{-\ell_{2}}, e^{-\ell_{3}}$.

I choose just to consider structures obtained by choosing the initial point to be $\mathbf{0}$ (resp. e), so that the group elements above are

$$
\alpha_{1} \cdot \alpha_{2} \cdot \alpha_{3} \cdots \alpha_{n}
$$

where $n$ is arbitrary, and each $\alpha_{i}, i=1, \ldots n$ is one of the generators $\mathbf{g}_{1} \equiv e^{\ell_{1}}$, $\mathbf{g}_{2} \equiv e^{\ell_{2}}, \mathbf{g}_{3} \equiv e^{\ell_{3}}$, or their inverses. The set of all elements of the form (87), without regard to any additional relations that may exist between the generators, is called the free group on the generators $\mathbf{g}_{1}, \mathbf{g}_{2}, \mathbf{g}_{3}$, and I denote it $\tilde{G}$.

Just as different choices of bases may generate a perfect lattice, different choices of generators may produce the same free group $\tilde{G}$. According to Magnus, Karrass and 
Solitar [12], the different choices of generators with this property are obtained from three types of operation:

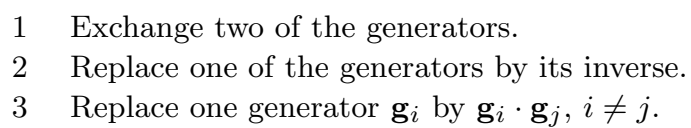

All different choices of generators are obtained by carrying out substitutions of types $1,2,3$, repeatedly a finite number of times.

Cermelli and Parry [9] have catalogued the crystal structures which arise in this process in the particular case of fields $\ell_{a}(\cdot)$, with $\ell_{a}(\mathbf{0})=\mathbf{e}_{a}$, with

$$
S=\lambda \mathbf{p} \otimes \mathbf{p},
$$

where $\lambda$ is rational and the components of $\mathbf{p}$ are relatively prime integers. It turns out that these structures are particular types of multilattice Cermelli and Parry[9], Pitteri and Zanzotto [13]. Here I describe how to find the point group symmetries of these structures, that derive from the operations (88) above. When (89) holds, according to Cermelli and Parry [9],

$$
\psi(\mathbf{x}, \mathbf{y})=\mathbf{x}+\mathbf{y}+\frac{1}{2} \lambda \mathbf{p}(\mathbf{p} \cdot \mathbf{x} \wedge \mathbf{y})
$$

and

$$
\ell_{a}(\mathbf{x})=\mathbf{e}_{a}+\frac{1}{2} \lambda \mathbf{p}\left(\mathbf{x} \wedge \mathbf{p} \cdot \mathbf{e}_{a}\right), \quad \mathbf{d}_{a}=\mathbf{e}_{a}+\frac{1}{2} \lambda p_{a}(\mathbf{p} \wedge \mathbf{x})
$$

where $\mathbf{p}$ has relatively prime components $p_{1}, p_{2}, p_{3} \in \mathbb{Z}$.

Consider the crystal structure which corresponds to flow along the three right invariant vector fields $\nu_{a b} \ell_{b}(\cdot)$, where $\ell_{b}(\cdot)$ is given by (91). Let $\nu_{a} \equiv \nu_{a b} \mathbf{e}_{b}$. One solves

$$
\dot{\mathbf{x}}=\nu_{a b} \boldsymbol{\ell}_{b}(\mathbf{x})=\nu_{a b} \mathbf{e}_{b}+\frac{1}{2} \lambda \mathbf{p}\left(\mathbf{x} \wedge \mathbf{p} \cdot \nu_{a b} \mathbf{e}_{b}\right)
$$

subject to $\mathbf{x}(0)=\mathbf{x}_{0}$, and calculates

$$
\mathbf{x}(1)=\exp \left(\nu_{a b} \ell_{b}\right)\left(\mathbf{x}_{0}\right)=\mathbf{x}_{0}+\nu_{a b} \ell_{b}\left(\mathbf{x}_{0}\right)
$$

SO

$$
e^{\nu_{a b} \ell_{b}}=\exp \left(\nu_{a b} \ell_{b}\right)(\mathbf{0})=\nu_{a b} \ell_{b}(0) \equiv \nu_{a}
$$

Therefore the coordinates of the generators of the free group $\tilde{G}$ which corresponds to these three vector fields are $\boldsymbol{\nu}_{1}, \boldsymbol{\nu}_{2}, \boldsymbol{\nu}_{3}$. Hence the operations which preserve the points that the elements of $\tilde{G}$ represent are of three types:

1. Exchange any two of $\boldsymbol{\nu}_{1}, \boldsymbol{\nu}_{2}, \boldsymbol{\nu}_{3}$, e.g. replace $\boldsymbol{\nu}_{1}, \boldsymbol{\nu}_{2}, \boldsymbol{\nu}_{3}$ by $\boldsymbol{\nu}_{2}, \boldsymbol{\nu}_{1}, \boldsymbol{\nu}_{3}$.

2. Replace one of $\boldsymbol{\nu}_{1}, \boldsymbol{\nu}_{2}, \boldsymbol{\nu}_{3}$, e.g. $\boldsymbol{\nu}_{1}$, by its inverse, e.g. $-\boldsymbol{\nu}_{1}$ (note that $\boldsymbol{\psi}\left(\boldsymbol{\nu}_{1},-\boldsymbol{\nu}_{1}\right)=$ 0).

3. Replace one of $\boldsymbol{\nu}_{1}, \boldsymbol{\nu}_{2}, \boldsymbol{\nu}_{3}$, e.g. $\boldsymbol{\nu}_{1}$, by $\boldsymbol{\psi}\left(\boldsymbol{\nu}_{1}, \boldsymbol{\nu}_{2}\right) \equiv \boldsymbol{\nu}_{1}+\boldsymbol{\nu}_{2}+\frac{1}{2} \lambda \mathbf{p}\left(\mathbf{p} \cdot \boldsymbol{\nu}_{1} \wedge \boldsymbol{\nu}_{2}\right)$, or by $\boldsymbol{\psi}\left(\boldsymbol{\nu}_{1}, \boldsymbol{\nu}_{3}\right)$.

Next one finds the vector fields $\mathbf{m}_{a}(\cdot)$ such that the new sets of generators are obtained by flow through unit time along $\mathbf{m}_{1}(\cdot), \mathbf{m}_{2}(\cdot), \mathbf{m}_{3}(\cdot)$.

1. For example, the fields $\mathbf{m}_{1} \equiv v_{2 b} \boldsymbol{\ell}_{b}, \mathbf{m}_{2} \equiv v_{1 b} \boldsymbol{\ell}_{b}, \mathbf{m}_{3} \equiv v_{3 b} \boldsymbol{\ell}_{b}$, are such that $e^{\mathbf{m}_{1}}=\boldsymbol{\nu}_{2}, e^{\mathbf{m}_{2}}=\boldsymbol{\nu}_{1}, e^{\mathbf{m}_{3}}=\boldsymbol{\nu}_{3}$. 
2. For example $\mathbf{m}_{1} \equiv-\nu_{1 b} \boldsymbol{\ell}_{b}, \mathbf{m}_{2} \equiv \nu_{2 b} \boldsymbol{\ell}_{b}, \mathbf{m}_{3} \equiv \nu_{3 b} \ell_{b}$ are such that $e^{\mathbf{m}_{1}}=-\boldsymbol{\nu}_{1}$, $e^{\mathbf{m}_{2}}=\boldsymbol{\nu}_{2}, e^{\mathbf{m}_{3}}=\boldsymbol{\nu}_{3}$,

3. Let $\boldsymbol{\kappa}_{1} \equiv \boldsymbol{\nu}_{1}+\boldsymbol{\nu}_{2}+\frac{1}{2} \lambda \mathbf{p}\left(\mathbf{p} \cdot \boldsymbol{\nu}_{1} \wedge \boldsymbol{\nu}_{2}\right), \mathbf{m}_{1} \equiv \kappa_{1 b} \ell_{b}, \mathbf{m}_{2} \equiv \nu_{2 b} \ell_{b}, \mathbf{m}_{3} \equiv \nu_{3 b} \ell_{b}$.

Then

$$
e^{\mathbf{m}_{1}}=\boldsymbol{\kappa}_{1}=\boldsymbol{\psi}\left(\boldsymbol{\nu}_{1}, \boldsymbol{\nu}_{2}\right), \quad e^{\mathbf{m}_{2}}=\boldsymbol{\nu}_{2}, \quad e^{\mathbf{m}_{3}}=\boldsymbol{\nu}_{3} .
$$

In this way, the new generators may be expressed as $e^{\mathbf{m}_{1}}, e^{\mathbf{m}_{2}}, e^{\mathbf{m}_{3}}$, where

$$
\mathbf{m}_{a}=\lambda_{a b}\left(\nu_{b c} \ell_{c}\right)
$$

and the matrix $\Lambda \equiv\left(\lambda_{a b}\right)$ is given, for example, by

$$
\begin{aligned}
\text { 1. } & \Lambda=\left(\begin{array}{lll}
0 & 1 & 0 \\
1 & 0 & 0 \\
0 & 0 & 1
\end{array}\right) \\
\text { 2. } \Lambda & =\left(\begin{array}{ccc}
-1 & 0 & 0 \\
0 & 1 & 0 \\
0 & 0 & 1
\end{array}\right) \\
\text { 3. } \Lambda & =\left(\begin{array}{ccc}
\kappa_{11} & \kappa_{12} & \kappa_{13} \\
0 & 1 & 0 \\
0 & 0 & 1
\end{array}\right)\left(\nu_{a b}\right)^{-1} \text {, where } \kappa_{1}=\left(\kappa_{1 a}\right) .
\end{aligned}
$$

Note that the dislocation density $S=\lambda \mathbf{p} \otimes \mathbf{p}$ derives from the fields $\boldsymbol{\ell}_{a}(\cdot)$, whereas the fields $\nu_{a b} \ell_{b}(\cdot)$ have dislocation density $\bar{\lambda} \overline{\mathbf{p}} \otimes \overline{\mathbf{p}}$, where $\bar{\lambda}=\lambda \operatorname{det}\left(\nu_{a b}\right), \overline{\mathbf{p}}=\left(\nu_{a b}\right)^{-T} \mathbf{p}$. One calculates that the expression for $\Lambda$ in 3 . may be reexpressed as

$$
\Lambda=\left(\begin{array}{ccc}
1+\frac{1}{2} \bar{\lambda} \bar{p}_{1} \bar{p}_{3} & 1+\frac{1}{2} \bar{\lambda} \bar{p}_{2} \bar{p}_{3} & \frac{1}{2} \bar{\lambda} \bar{p}_{3}^{2} \\
0 & 1 & 0 \\
0 & 0 & 1
\end{array}\right),
$$

which shows that the matrices $\Lambda$ depend just on the appropriate dislocation density tensor.

Also note that, in the particular case when the dislocation density is zero, $\bar{\lambda} \overline{\mathbf{p}}=\mathbf{0}$ and the last form of $\Lambda$ is

$$
\Lambda=\left(\begin{array}{lll}
1 & 1 & 0 \\
0 & 1 & 0 \\
0 & 0 & 1
\end{array}\right)
$$

In this particular case, then, repeated substitutions of types 1., 2., 3., which produce arbitrary products of

$$
\left(\begin{array}{lll}
0 & 1 & 0 \\
1 & 0 & 0 \\
0 & 0 & 1
\end{array}\right),\left(\begin{array}{ccc}
-1 & 0 & 0 \\
0 & 1 & 0 \\
0 & 0 & 1
\end{array}\right),\left(\begin{array}{lll}
1 & 1 & 0 \\
0 & 1 & 0 \\
0 & 0 & 1
\end{array}\right),
$$

and their inverses, generate the unimodular group $G L_{3}(\mathbb{Z})$, and one recovers the statement that surrounds (2).

Now, returning to the case where $S \neq 0$, let us calculate which of these changes of generators produces rotations of the crystal structure. To begin with confine attention 
to the case $\left(\nu_{a b}\right)=$ id., $\ell_{a}(\mathbf{0})=\mathbf{e}_{a}$, and consider equation (71): choose $\tilde{L}$, in (71), to be any product of the three types of matrix $\Lambda$, above namely

$$
\left(\begin{array}{lll}
0 & 1 & 0 \\
1 & 0 & 0 \\
0 & 0 & 1
\end{array}\right),\left(\begin{array}{ccc}
-1 & 0 & 0 \\
0 & 1 & 0 \\
0 & 0 & 1
\end{array}\right),\left(\begin{array}{ccc}
\kappa_{11} & \kappa_{12} & \kappa_{13} \\
0 & 1 & 0 \\
0 & 0 & 1
\end{array}\right),
$$

and their inverses. The right hand side of $(71)$ is $\tilde{L}_{b a} \ell_{b}\left(C^{\prime}, L \mathbf{x}\right)$, where Pontryagin's canonical fields $\ell_{a}\left(C^{\prime}, \cdot\right)$ satisfy $\ell_{a}\left(C^{\prime}, \mathbf{0}\right)=\mathbf{e}_{a}$. Thus the right hand side of $(71)$ is right invariant with respect to the same composition function as the basis fields $\ell_{a}\left(C^{\prime}, \cdot\right)$. Define $\tilde{\ell}_{a}(\tilde{C}, \cdot)$, (where $\tilde{C}$ will be specified below), by

$$
\tilde{\ell}_{a}(\tilde{C}, \tilde{L} \mathbf{x}) \equiv \tilde{L} \boldsymbol{\ell}_{a}(C, \mathbf{x}),
$$

that is, define $\tilde{\ell}_{a}(\tilde{C}, \cdot)$ to be the right invariant field obtained by elastic deformation, through (homogeneous) deformation gradient $\tilde{L}$, of $\ell_{a}(C, \cdot)$. The fields $\ell_{a}(C, \cdot)$ have constant dislocation density, which equals the constant dislocation density of $\tilde{\ell}_{a}(\tilde{C}, \cdot)$. This implies, by an analogue of (53), that

$$
\tilde{C}_{i j k} \tilde{L}_{j p} \tilde{L}_{k q}=\tilde{L}_{i r} C_{r p q},
$$

independently of the values of $\ell_{a}(C, 0)$, in fact. This defines $\tilde{C}$. So from (63)

$$
\tilde{C}=C^{\prime} .
$$

Hence (71) may be written as

$$
\tilde{\ell}_{a}\left(C^{\prime}, \cdot\right)=\tilde{L}_{b a} \ell_{b}\left(C^{\prime}, \cdot\right)
$$

which turns out to be a useful version of (8), in this context. (Note that the dislocation density of the fields on the left of (98) may be denoted $S$, where $\varepsilon_{j k \ell} S_{i \ell}=C_{i j k}$. The fields on the right of (98) have dislocation density $\tilde{L}^{-1} S^{\prime} \tilde{L}^{-T} \operatorname{det} L$, when the relation between $S^{\prime}$ and $C^{\prime}$ is $\varepsilon_{j k \ell} S_{i \ell}^{\prime}=C_{i j k}^{\prime}$. One may verify that $S=\tilde{L}^{-1} S^{\prime} \tilde{L}^{-T} \operatorname{det} L$, by virtue of the connection (63), between $C^{\prime}$ and $C$.)

Equation (98) is a connection between right invariant fields in the Lie algebra of vector fields with structure constants $C^{\prime}$, and elastic deformation of basis fields in that algebra, just as (8) is a connection between a change of basis vectors and an elastic (homogeneous) deformation of those vectors. In fact, (98) holds independently of the particular choice of $\tilde{L}$ that was specified above.

Now recall that $\tilde{L}$ has been chosen so that the fields $\tilde{L}_{b a} \ell_{b}\left(C^{\prime}, \cdot\right)$ generate the same set of points as the fields $\ell_{b}\left(C^{\prime}, \cdot\right)$. If $\tilde{L}$ is orthogonal, this new set of fields is that obtained from the original set by (elastic) rotation. But the iteration process described above commutes with elastic deformation: to see this consider the effect of an arbitrary elastic deformation $\boldsymbol{\theta}: \mathbf{x} \rightarrow \theta(\mathbf{x})$ on the flow corresponding to a vector field $\boldsymbol{\ell}(\cdot)$. So let $\tilde{\ell}(\boldsymbol{\theta}(\mathbf{x})) \equiv \nabla \boldsymbol{\theta}(\mathbf{x}) \boldsymbol{\ell}(\mathbf{x})$, and note that the unique solution of

$$
\dot{\mathbf{y}}=\tilde{\ell}(\mathbf{y}), \quad \mathbf{y}(0)=\boldsymbol{\theta}\left(\mathbf{x}_{0}\right),
$$

is $\mathbf{y}(t)=\boldsymbol{\theta}(\mathbf{x}(t))$, where $\mathbf{x}(t)$ solves

$$
\dot{\mathbf{x}}=\ell(\mathbf{x}), \quad \mathbf{x}(0)=\mathbf{x}_{0} .
$$


So, recalling also that $(\exp \ell)(\cdot)$ denotes the map which represents 'flow through unit time along the vector field $\ell(\cdot)$, i.e. $(\exp \ell)\left(\mathbf{x}_{0}\right)=\mathbf{x}(1)$, it follows that

$$
(\exp \tilde{\boldsymbol{\ell}})(\boldsymbol{\theta}(\mathbf{x}))=\boldsymbol{\theta}(\exp \boldsymbol{\ell}(\mathbf{x})) .
$$

Therefore, the set of points obtained by iteration, using the fields $\tilde{\ell}_{a}(C, \cdot)$ equals the elastic image (via homogeneous elastic deformation $\tilde{L}$ ) of the set of points obtained by iteration using the fields $\ell_{a}(C, \cdot)$. Thus the orthogonal $\tilde{L}$, of the form given above, are elements of the point group of the structure.

For example, if $S=2 \mathbf{e}_{1} \otimes \mathbf{e}_{1}$, the matrices $\Lambda$ of type 3. (i.e. $\kappa$ and their inverses) are

$$
\left(\begin{array}{lll}
1 & 1 & 0 \\
0 & 1 & 0 \\
0 & 0 & 1
\end{array}\right),\left(\begin{array}{lll}
1 & 0 & 1 \\
0 & 1 & 0 \\
0 & 0 & 1
\end{array}\right),\left(\begin{array}{lll}
1 & 0 & 0 \\
1 & 1 & 0 \\
0 & 0 & 1
\end{array}\right),\left(\begin{array}{lll}
1 & 0 & 0 \\
1 & 1 & 1 \\
0 & 0 & 1
\end{array}\right),\left(\begin{array}{lll}
1 & 0 & 0 \\
0 & 1 & 0 \\
1 & 0 & 1
\end{array}\right),\left(\begin{array}{ccc}
1 & 0 & 0 \\
0 & 1 & 0 \\
-1 & 1 & 1
\end{array}\right),
$$

and their inverses. Together with the matrices of types 1. and 2., these are elements of $G L_{3}(\mathbb{Z})$, and the list includes a set of generators of that group. Hence the point group of the structure is the set of orthogonal elements of $G L_{3}(\mathbb{Z})$, it is the cubic group.

Finally, in this section, in order to prepare for a discussion of the symmetry of strain energy functions which have symmetries appropriate to the structures which have been constructed by the iteration process, I discuss the 'rotational symmetries' of the fields $\tilde{\ell}_{c}\left(C^{\prime}, \cdot\right)$ in $(74)((74)$ is the generalization of $(95)$, or $(71)$, to the case where the fields involved are arbitrary right invariant fields, satisfying $\left.\tilde{\ell}_{a}(\mathbf{0})=\nu_{a b} \mathbf{e}_{b}=\boldsymbol{\nu}_{a}\right)$. So I adapt the notation of $(74)$, put $\ell_{a}^{+}\left(C^{\prime}, \tilde{L} \mathbf{x}\right)=\tilde{L} \ell_{a}(C, \mathbf{x})$ and rewrite $(74)$ as

$$
\tilde{L} \tilde{\ell}_{c}(C, \mathbf{x})=\boldsymbol{\ell}_{c}^{+}\left(C^{\prime}, \tilde{L} \mathbf{x}\right)=\tilde{\gamma}_{c d} \tilde{\ell}_{d}\left(C^{\prime}, \tilde{L} \mathbf{x}\right)
$$

where $\tilde{\gamma}=\nu \tilde{L}^{T} \nu^{-1}, \nu=\left(\nu_{a b}\right)$. Observations similar to those above apply to (102): if $\tilde{\gamma}$ is chosen so that $\tilde{\gamma}_{c d} \tilde{\ell}_{d}\left(C^{\prime}, \cdot\right)$ generates the same set of points as $\tilde{\ell}_{c}\left(C^{\prime}, \cdot\right)$, and if $\tilde{L}$ is orthogonal, this new set of points is obtained from the original set by (elastic) rotation. The orthogonal matrices so chosen are elements of the point group of the structure. The corresponding matrices $\tilde{\gamma}$ are products of the three types of matrix $\Lambda$ listed above, together with their inverses, noting that the third type of matrix $\Lambda$, above, depends just on the dislocation density corresponding to the fields $\tilde{\ell}_{c}\left(C^{\prime}, \cdot\right)$.

Also, in generalization of the parenthetical remarks which follow (98), note that if one chooses $C$ via (53), when $S$ and $\tilde{\ell}_{r}(\mathbf{0})$ are given, then the fields $\tilde{\ell}_{r}(C, \cdot)$ have dislocation density $S$, as do the fields $\ell_{r}^{+}\left(C^{\prime}, \cdot\right)$. The fields on the right hand side of (102), namely $\tilde{\gamma}_{c d} \tilde{\ell}_{d}\left(C^{\prime}, \cdot\right)$ thus have dislocation density $\tilde{\gamma}^{-T} S^{\prime} \tilde{\gamma}^{-1} \operatorname{det}(\tilde{\gamma})$ (where $S^{\prime}$ is related to $C^{\prime}$ by the analogue of $(53)$, where $\tilde{\boldsymbol{\ell}}_{c}\left(C^{\prime}, \mathbf{0}\right)$ satisfies $\tilde{L} \boldsymbol{\nu}_{c} \equiv \tilde{L} \boldsymbol{\ell}_{c}(C, \mathbf{0})=$ $\tilde{\gamma}_{c d} \tilde{\ell}_{d}\left(C^{\prime}, \mathbf{0}\right)$ from (102), and where $C^{\prime}$ is related to $C$ via $\left.(63)\right)$. One may check that $S=\tilde{\gamma}^{-T} S^{\prime} \tilde{\gamma}^{-1} \operatorname{det}(\tilde{\gamma})$.

\section{Global and point group symmetry of strain energy functions}

I consider the generalization of (11)-(13) to the case when the energy function depends on the dislocation density as well as on values $\nu_{a}$, say, of some lattice vector fields $\boldsymbol{\ell}_{a}(\cdot)$. 
When the dislocation density is non zero, the lattice vector fields are non-constant, so I define fields associated with the values $\nu_{a}, S$ which are the arguments of an energy function

$$
w=w\left(\left\{\boldsymbol{\nu}_{a}\right\}, S\right)
$$

in the following way: let $\boldsymbol{\nu}_{a}$ be the values of fields $\boldsymbol{\ell}_{a}(\cdot)$ (which are to be determined) at the origin. Given the values $\ell_{a}(\mathbf{0})$, and $S$, define structure constants $C$ via (53). Construct the basis (Pontryagin) right invariant fields corresponding to $C$ according to section 2 (vi), and let $\ell_{a}(\cdot)$ be the right invariant fields in this coordinate system which are such that $\boldsymbol{\ell}_{a}(\mathbf{0})=\boldsymbol{\nu}_{a}$. Then the fields $\boldsymbol{\ell}_{a}(\cdot)$ are uniquely determined by the quantities $\left\{\boldsymbol{\nu}_{a}\right\}$ and $S$, and they have dislocation density $S$.

The fields $\ell_{a}(\cdot)$ determine a set of points, by the iterative process outlined above, which is intimately associated with the continuum strain energy (103), just as the energy of a perfect crystal continuum is associated with the lattice of points $L$ in definition (1). I take the point of view that the symmetries of the set of points generated by the iterative process should be adopted as symmetries of the corresponding energy density, just as symmetries of the lattice $L$ are adopted as symmetries of the energy (11). Symmetries of the set of points obtained by the iterative process have been discussed above: on the one hand, there are the changes of generators which preserve the set of points (analogous to the changes of basis of lattice $L$ ), and on the other hand there are the elastic rotations which map the set of points to itself (analogous to the point group symmetries of the lattice). In the perfect crystal case, the 'global' symmetries of the lattice are represented by the matrices $\gamma=\left(\gamma_{a b}\right) \in G L_{3}(\mathbb{Z})$ - they are such that

$$
w\left(\left\{\mathbf{e}_{a}\right\}\right)=w\left(\left\{\gamma_{a b} \mathbf{e}_{b}\right\}\right) .
$$

In the defective crystal case, where $S, C$ and $\nu_{a} \equiv \boldsymbol{\ell}_{a}(0)$ are related via (53), the lattice vector fields associated with values $\left\{\boldsymbol{\nu}_{a}\right\}, S$ are denoted $\tilde{\boldsymbol{\ell}}_{c}(C, \cdot)$, and they have dislocation density $S$. The fields which produce a change in generators are of the form $\tilde{\gamma}_{c d} \tilde{\ell}_{d}(C, \cdot)$, with dislocation density $(\tilde{\gamma})^{-T} S(\tilde{\gamma})^{-1} \operatorname{det}(\tilde{\gamma})$, taking the values $\tilde{\gamma}_{c d} \boldsymbol{\nu}_{d}$ at the origin.

Put

$$
S_{\tilde{\gamma}}=\tilde{\gamma}^{-T} S \tilde{\gamma}^{-1} \operatorname{det}(\tilde{\gamma}) .
$$

Then the global symmetries of an energy function of form (103) may be expressed as

$$
w\left(\left\{\boldsymbol{\nu}_{a}\right\}, S\right)=w\left(\left\{\tilde{\gamma}_{a b} \boldsymbol{\nu}_{b}\right\}, S_{\tilde{\gamma}}\right),
$$

and this is the required generalization of (104) to the case of defective crystals. Note that in the case where $S=\lambda \mathbf{p} \otimes \mathbf{p}$, it has been shown that $\tilde{\gamma}$ depends solely on $S$, and the allowed forms of $\tilde{\gamma}$ have been given explicitly.

Next, one accepts that

$$
w\left(\left\{\boldsymbol{\nu}_{a}\right\}, S\right)=w\left(\left\{R \nu_{a}\right\}, S\right)
$$

where $R$ is an arbitrary orthogonal transformation, for the following reason. As before, let $\tilde{\boldsymbol{\ell}}_{a}(C, \mathbf{0}), C, S$ be related by (53), with $\boldsymbol{\ell}_{a}(C, \mathbf{0})=\boldsymbol{\nu}_{a}$ denoted $\boldsymbol{\ell}_{a}(\mathbf{0})$ in (53). The fields $\ell(C, \cdot)$ generate a set of points $\Sigma$, say, by the iteration process described in section 3. Fields $\ell_{a}^{+}\left(C^{\prime}, \cdot\right)$ defined by $\ell_{a}^{+}\left(C^{\prime}, R \mathbf{x}\right)=R \tilde{\ell}_{a}(C, \mathbf{x})$ represent fields obtained from 
$\tilde{\ell}_{a}(C, \cdot)$ by (orthogonal) elastic deformation, so these fields have dislocation density $S$, since the dislocation density is invariant under elastic deformation. (Note that $C$ and $C^{\prime}$ are related by an analogue of (96)). Moreover $\ell_{a}^{+}\left(C^{\prime}, \mathbf{0}\right)=R \boldsymbol{\nu}_{a}, a=1,2,3$. According to $(102)_{2}$, with $\tilde{L} \equiv R$, the fields $\ell_{a}^{+}\left(C^{\prime}, \cdot\right)$ are right invariant fields with respect to the composition function which has structure constants $C^{\prime}$. One checks that the value of $C^{\prime}$ defined by the analogue of (96) is indeed such that $\boldsymbol{\ell}_{a}^{+}\left(C^{\prime}, \cdot\right)=R \boldsymbol{\nu}_{a}$, $S, C^{\prime}$ satisfy (53). It follows that the fields $\ell_{a}^{+}\left(C^{\prime}, \cdot\right)$ are those to be used in the iteration procedure, to determine the set of points associated with values $\left\{R \boldsymbol{\nu}_{a}\right\}, S$. So, by the commutation property discussed above, the set of points associated with the left side of (107) is a rotation of the set of points associated with the right side of (107). Therefore (107) expresses the invariance of the interaction energy of a set of points with respect to rigid rotation, and in this guise (107) is an acceptable hypothesis.

Finally, to discuss the point group symmetries, one confines attention, in (106), to the particular changes of generators which produce rotation of the set of points $\Sigma$. According to (102), these changes of generators are represented by matrices $\tilde{\gamma}$ such that

$$
\nu \tilde{L}^{T}=\tilde{\gamma} \nu, \quad \text { or } \quad \tilde{L} \boldsymbol{\nu}_{a}=\tilde{\gamma}_{a b} \boldsymbol{\nu}_{b}
$$

where $\tilde{L}$ is orthogonal. Let the set of orthogonal matrices $\tilde{L}$ such that $(108)_{2}$ has a solution for some appropriate $\tilde{\gamma} \equiv\left(\gamma_{a b}\right)$ be called the point group of the defective crystal, denoted $P_{d}$. Let the set of matrices $\tilde{\gamma}$ such that $(108)_{2}$ has a solution for some $\tilde{L} \in P_{d}$ be called the lattice group of the defective crystal, denoted $\tilde{L}_{d}$. Note that $(108)_{2}$ is the analogue of (3). Further, by analogy with the procedure, that was recalled in the introduction, for perfect crystals, let $C_{d}$ be the matrix with entries $\boldsymbol{\nu}_{a} \cdot \boldsymbol{\nu}_{b}$. Then (107) implies that $w$ may be written as

$$
w=\tilde{w}\left(C_{d}, S\right),
$$

and that is the analogue of (13), in the introduction.

From $(108)_{2}$

Since (106) may be written as

$$
C_{d}=\tilde{\gamma} C_{d} \tilde{\gamma}^{T}, \quad \tilde{\gamma} \in \tilde{L}_{d} .
$$

$$
\tilde{w}\left(C_{d}, S\right)=\tilde{w}\left(\tilde{\gamma} C_{d} \tilde{\gamma}^{T}, S_{\tilde{\gamma}}\right)
$$

for the allowed forms of $\tilde{\gamma}$ discussed in section 3, it follows from (110) that

$$
\tilde{w}\left(C_{d}, S\right)=\tilde{w}\left(C_{d}, S_{\tilde{\gamma}}\right), \quad \tilde{\gamma} \in \tilde{L}_{d} .
$$

This equation shows that the dependence of the energy function on dislocation density is constrained by symmetry properties of the set of points chosen to represent the local structure of the defective crystal.

Further work will consider, in particular, how $\tilde{L}, \tilde{\gamma}$ are determined in detail, for choices of $S$ other than $S=\lambda \mathbf{p} \otimes \mathbf{p}$, and investigate corresponding representations of the functions $\tilde{w}$ which satisfy (111), (112). Also, note that I have not discussed, for perfect crystals or defective crystals, how the symmetry properties of the energy functions above transfer to symmetry properties of strain energy functions (based on a given reference configuration) in nonlinear elasticity theory. For perfect crystals, one may refer to Pitteri and Zanzotto [13] to see how this can be done - it is another avenue for exploration, in the case of defective crystals.

Acknowledgements I thank the two referees for helpful comments. 


\section{References}

1. C. Davini, A proposal for a continuum theory of defective crystals. Arch. Rational Mech. Anal., 96, 295-317 (1986)

2. L. S. Pontryagin, Topological groups (2nd Edition), Gordon and Breach, New York, London, Paris (1955)

3. P. J. Olver, Equivalence, invariants, and symmetry. Cambridge University Press, Cambridge (1996)

4. V. S. Varadarajan, Lie groups, Lie algebras, and their representations, Prentice-Hall, Inc., Englewood Cliffs, N.J. (1974)

5. F. W. Warner, Foundations of Differentiable Manifolds and Lie Groups, Springer, New York (1983)

6. J. G. F. Belinfante, Lie algebras and inhomogeneous simple materials, SIAM J. Appl. Math. 25 260-268 (1973)

7. B. A. Bilby, Geometry and Continuum Mechanics, reprint.

8. G. P. Parry, Group properties of defective crystal structures Mathematics and Mechanics of Solids 8, 515-538 (2003)

9. P. Cermelli and G. P. Parry, The structure of uniform discrete defective crystals, Continuum Mechanics and Thermodynamics, 18 (1-2), 47-61 (2006)

10. M. Elzanowksi and G. P. Parry, Material symmetry in a theory of continuously defective crystals, J. Elasticity 74(3), 215-237 (2004)

11. C. Caratheodory, Calculus of Variations and Partial Differential Equations of the First Order, Part I, Holden-Day, Inc., San Francisco, London, Amsterdam (1965)

12. W. Magnus, A. Karrass and D. Solitar, Combinatorial group theory, Dover Publications, Inc., Mineola, New York (1976)

13. M. Pitteri and G. Zanzotto, Continuum models for phase transitions and twinning in crystals, Chapman and Hall/CRC, Boca Raton, London, New York, Washington D.C. (2003) 